\title{
Hyers-Ulam stability and existence criteria for coupled fractional differential equations involving $p$-Laplacian operator
}

\author{
Hasib Khan ${ }^{1,2}$, Wen Chen ${ }^{1 *}$, Aziz Khan ${ }^{3 *}$ (I), Tahir S. Khan ${ }^{3}$ and Qasem M. Al-Madlal ${ }^{4}$
}

\author{
"Correspondence: \\ chenwen@hhu.edu.cn; \\ azizkhan927@yahoo.com \\ ${ }^{1}$ College of Engineering Mechanics \\ and Materials, Hohai University, \\ Nanjing, P.R. China \\ ${ }^{3}$ University of Peshawar, Peshawar, \\ Pakistan \\ Full list of author information is \\ available at the end of the article
}

\begin{abstract}
In this article, by using nonlinear Leray-Schauder-type alternative and Banach's fixed point theorem, we investigate existence and uniqueness of solutions. We also prove Hyers-Ulam stability for the proposed coupled system of fractional differential equations (FDEs) with the nonlinear $p$-Laplacian operator and Riemann-Liouville integral boundary conditions (IBCs). An illustrative example is presented to demonstrate our main results.
\end{abstract}

Keywords: Fractional differential equations; Riemann-Liouville integral boundary conditions; $p$-Laplacian operator; Hyers-Ulam stability; Banach's fixed point theorem

\section{Springer}

\section{Introduction}

Fractional calculus is a generalization of classical calculus. FDEs are proved to be valuable tools compared to integer order differential equations. Different physical and natural phenomena were modeled using FDEs in various disciplines, such as combustion theory, papulation biology, non-Newtonian mechanics, control theory, aerodynamics, hydro- and electro-dynamics, economics, bioengineering, networking, system of MongeKantorovich partial differential equations, image and signal processing, viscoelasticity, blood flow, game theory, chemistry; for details we refer to [1-10].

Recently, differential equations involving the nonlinear $p$-Laplacian operator gained attention from researchers. Articles dealing with ordinary differential equations and partial differential equations involving the nonlinear $p$-Laplacian operator have been studied. In the last few years, the mentioned area was extended to FDEs using different fractional order integrals and differential operators. By different mathematical approaches FDEs were investigated for existence and uniqueness of solutions, as well as for multiple positive solutions [11-20].

Further, FDEs involving the nonlinear $p$-Laplacian operator with integral boundary conditions have attracted researchers of various disciplines, because such systems are increasingly used in modeling [21-24]. Zhi et al. [14] have studied FDEs with the $p$-Laplacian nonlinear operator aiming to show existence of positive solutions for a nonlocal boundary value problem and have given a valuable example to demonstrate the results. The problem

(c) The Author(s) 2018. This article is distributed under the terms of the Creative Commons Attribution 4.0 International License (http://creativecommons.org/licenses/by/4.0/), which permits unrestricted use, distribution, and reproduction in any medium, provided you give appropriate credit to the original author(s) and the source, provide a link to the Creative Commons license, and indicate if changes were made. 
is given by

$$
\begin{aligned}
& \left(\varphi_{p}\left(\mathcal{D}^{\theta_{1}} \omega(x)\right)\right)^{\prime \prime}=\mathcal{F}\left(x, \omega(x), \mathcal{D}^{\theta_{2}} \omega(x)\right), \quad x \in(0,1), \\
& \left.\omega(x)\right|_{x=0}=\left.\omega^{\prime \prime}(x)\right|_{x=0}=0, \quad \omega(1)=\int_{0}^{1} g(\theta) \omega(\theta) d \theta, \\
& \left(\varphi_{p}\left(\mathcal{D}^{\theta_{1}}\right) \omega(0)\right)^{\prime}=\xi_{1}\left(\varphi_{p}\left(\mathcal{D}^{\theta_{1}}\right) \omega\left(a_{1}\right)\right)^{\prime}, \\
& \varphi_{p}\left(\mathcal{D}^{\theta_{1}}\right) \omega(1)=\xi_{2}\left(\varphi_{p}\left(\mathcal{D}^{\theta_{1}}\right) \omega\left(b_{2}\right)\right),
\end{aligned}
$$

where $\mathcal{D}^{\theta_{1}}, \mathcal{D}^{\theta_{2}}$ represent derivatives of order $\theta_{1}, \theta_{2}$ in Caputo sense, where $2<\theta_{1} \leq 3,1<$ $\theta_{2}<\theta_{1}-1<2,0 \leq \xi_{1}, \xi_{2}<1,0<a_{1} \leq b_{2}<1$, and $\varphi_{p}$ is the nonlinear $p$-Laplacian operator. $\mathrm{Hu}$ and Zhang [25] investigated a coupled system of FDEs with the nonlinear $p$-Laplacian operator and infinite boundary conditions, namely the following problem:

$$
\begin{aligned}
& \mathcal{D}^{\theta_{1}^{*}} \varphi_{p}\left(\mathcal{D}^{\theta_{1}} \omega_{1}(r)\right)=h\left(r, \omega_{2}(r), \mathcal{D}^{\theta_{2}-1}, \mathcal{D}^{\theta_{2}-1} \omega_{2}(r), \ldots, \mathcal{D}^{\theta_{2}-(n-1)} \omega_{2}(r)\right), \quad r \in(0,1), \\
& \mathcal{D}_{2}^{\theta_{2}^{*}} \varphi_{p}\left(\mathcal{D}^{\theta_{2}} y(r)\right)=g\left(r, \omega_{1}(r), \mathcal{D}^{\theta_{1}-1}, \mathcal{D}^{\theta_{1}-1} \omega_{1}(r), \ldots, \mathcal{D}^{\theta_{1}-(n-1)} \omega_{1}(r)\right), \quad r \in(0,1), \\
& \omega_{1}^{\prime}(0)=\cdots=\omega_{1}^{(n-1)}(0)=\mathcal{D}^{\theta_{1}} \omega_{1}(0)=0, \quad \omega_{1}(0)=\sum_{i=1}^{\infty} a_{i} \omega_{1}\left(\mu_{i}\right), \\
& \omega_{2}^{\prime}(0)=\cdots=\omega_{2}^{(n-1)}(0)=\mathcal{D}^{\theta_{2}} \omega_{2}(0)=0, \quad \omega_{2}(0)=\sum_{i=1}^{\infty} b_{i} \omega_{2}\left(v_{i}\right),
\end{aligned}
$$

where $\mathcal{D}^{\theta_{i}^{*}}, \mathcal{D}^{\theta_{i}}$ for $i=1,2$, are Caputo fractional derivatives, $0<\theta_{1}^{*}, \theta_{2}^{*}<1, n-1<\theta_{1}, \theta_{2}<n$, $0<\mu_{1}<\mu_{2}<\cdots<\mu_{i}<\cdots<1,0<v_{1}<v_{2}<\cdots<v_{i}<\cdots<1, \sum_{i=1}^{\infty}\left|a_{i}\right|<\infty, \sum_{i=1}^{\infty}\left|b_{i}\right|<\infty$, $\sum_{i=1}^{\infty} a_{i}=\sum_{i=1}^{\infty} b_{i}=1$, and $h, g$ are real-valued continuous functions.

Recently, Ali et al. [26] investigated a coupled system of fractional differential equations with noninteger order integral boundary conditions for the existence and uniqueness of solutions, and furthermore, checked Hyers-Ulam stability. By using the topological degree theory, some special conditions were developed to show stability. As an application, an expressive example was provided to demonstrate the considered problem, which is given below:

$$
\begin{aligned}
& \mathcal{D}^{\theta_{1}} \omega_{1}(r)-f\left(r, \omega_{2}(r)\right)=0, \quad r \in[0,1], \\
& \mathcal{D}^{\theta_{2}} \omega_{2}(r)-g\left(r, \omega_{1}(r)\right)=0, \quad r \in[0,1], \\
& \omega_{1}(0)=0,\left.\quad \omega_{1}(r)\right|_{t=1}=\frac{1}{\Gamma(\gamma)} \int_{0}^{T}(r-s)^{\gamma-1} p\left(\omega_{1},(s)\right) d s, \\
& \omega_{2}(0)=0,\left.\quad \omega_{2}(r)\right|_{t=1}=\frac{1}{\Gamma(\delta)} \int_{0}^{T}(r-s)^{\delta-1} q\left(\omega_{2}(s)\right) d s,
\end{aligned}
$$

where $\mathcal{D}^{\theta_{1}}, \mathcal{D}^{\theta_{2}}$ are Caputo fractional derivatives, $\theta_{1}, \theta_{2}, \gamma, \delta \in(1,2], p, q \in L[0,1]$ and $f, g \in J \times \mathcal{R} \rightarrow \mathcal{R}$, the functions involved in fractional IBCs are continuous and also satisfy certain growth conditions.

Inspired by the aforementioned work, we investigate existence and uniqueness of solutions and prove Hyres-Ulam stability for the coupled suggested problem FDEs involving 
the nonlinear $p$-Laplacian operator with integral boundary conditions, namely

$$
\begin{array}{ll}
\left.\mathcal{D}_{1}^{\theta_{1}^{*}} \varphi_{p}\left(\mathcal{D}^{\theta_{1}} \omega_{1}(r)\right)=f\left(r, \omega_{1}(r), \omega_{2}(r)\right)\right), & r \in[0,1], \\
\left.\mathcal{D}^{\theta_{2}^{*}} \varphi_{p}\left(\mathcal{D}^{\theta_{2}} \omega_{2}(r)\right)=g\left(r, \omega_{1}(r), \omega_{2}(r)\right)\right), & r \in[0,1], \\
\left.\varphi_{p}\left(\mathcal{D}^{\theta_{1}} \omega_{1}(r)\right)\right|_{r=0}=\left.\omega_{1}^{\prime}(r)\right|_{r=0}=0, & \left.\omega_{1}(r)\right|_{r=0}=\gamma I^{\alpha-1} \omega_{1}(\eta), \\
\left.\varphi_{p}\left(\mathcal{D}^{\theta_{2}} \omega_{2}(r)\right)\right|_{r=0}=\left.\omega_{2}^{\prime}(r)\right|_{r=0}=0, & \left.\omega_{2}(r)\right|_{r=0}=\delta I^{\beta-1} \omega_{2}(\xi),
\end{array}
$$

where $\mathcal{D}^{\theta_{i}}, \mathcal{D}_{i}^{\theta_{i}^{*}}$ for $i=1,2$ are Caputo fractional derivatives, $\theta_{i} \in(1,2], \theta_{i}^{*} \in(0,1], f, g \in$ $C\left([0,1] \times \mathcal{R}^{2}, \mathcal{R}\right), i=1,2$ are continuous functions and $\alpha, \beta \geq 1, \gamma, \delta \in[-1,0] \varphi_{p}(\vartheta)=$ $|\vartheta|^{p-2} \vartheta$ is the $p$-Laplacian operator where $1 / p+1 / q=1, \varphi_{q}$ denotes the inverse of $p$-Laplacian. For existence and uniqueness of solutions, employing nonlinear LeraySchauder-type alternative and Banach's fixed point theorem, new results are obtained for the coupled considered FDEs involving the nonlinear $p$-Laplacian operator with IBCs. The important aspect of this article is to check stability for the coupled considered FDEs involving the nonlinear $p$-Laplacian operator. In the literature, different types of stability were presented for functional, differential and integral equations, for example, Lyapunov and exponential stability $[27,28]$. But checking such stability is difficult and time consuming due to calculation of Lyapunov functions. An interesting and motivating stability method was introduced by Ulam and then by Hyers in 1941, which is known as HyersUlam stability $[29,30]$. Such stability has outstanding applications in integer order and fractional order differential equations appearing in physics, optimization, numerical analysis, biological phenomena, economic, biochemistry, etc. For the details of using HyresUlam stability, we suggest [9,31-33]. In the following sections, we provide necessary definitions, lemmas, assumptions, as well as decribe the stability method and an example for the coupled considered FDEs with the nonlinear $p$-Laplacian operator with integral boundary conditions.

\section{Background material and auxiliary results}

Let us introduce $\mathcal{X}=\left\{\omega_{1}(r) \mid \omega_{1}(r) \in \mathcal{C}^{1}([0,1])\right\}$ as the space all continuous functions, endowed with a norm $\left\|\omega_{1}\right\|=\max \left\{\omega_{1}(r), r \in[0,1]\right\}$, here $(\mathcal{X},\|\cdot\|)$ is obviously a Banach space, let $\mathcal{Y}=\left\{\omega_{2}(r) \mid \omega_{2}(r) \in \mathcal{C}^{1}\right\}$. Then the product space denoted by $\left(\mathcal{X} \times \mathcal{Y},\left\|\left(\omega_{1}, \omega_{2}\right)\right\|\right)$, equipped with the norm $\left\|\left(\omega_{1}, \omega_{2}\right)\right\|=\left\|\omega_{1}\right\|+\left\|\omega_{2}\right\|$, is also a Banach space. This will be used throughout in the considered coupled FDEs with the nonlinear $p$-Laplacian with IBCs. Now recall the following definition which can be traced to [34-36].

Definition 2.1 Let $\digamma$ be a given function on a closed interval $[0, b]$. Then the non-integer order derivative in the Caputo sense of $\digamma$ is defined by

$$
\mathcal{D}^{\theta_{1}} \digamma(r)=\int_{0}^{r} \frac{(r-\tau)^{n-\theta_{1}-1}}{\Gamma\left(n-\theta_{1}\right)}\left(\frac{d^{n}}{d \tau^{n}} \digamma(\tau)\right) d \tau, \quad \theta_{1} \in(n-1, n],
$$

where $n-1=\left[\theta_{1}\right]$. In particular, if $\digamma$ is defined on the interval $[0, b]$ and $\theta_{1} \in(0,1)$, then

$$
\mathcal{D}^{\theta_{1}} \digamma(r)=\frac{1}{\Gamma\left(1-\theta_{1}\right)} \int_{0}^{r} \frac{\digamma^{\prime}(\tau)}{(r-\tau)^{\theta_{1}}} d \tau, \quad \text { where } \varphi^{\prime}(s)=\frac{d \varphi(s)}{d s} .
$$

It is to be noted that the integral on the right-hand side is pointwise defined on $\mathcal{R}^{+}$. 
Definition 2.2 The integral of arbitrary order $\theta_{1} \in \mathcal{R}^{+}$in the Riemann-Liouville sense for a function $\varphi: \mathcal{R}^{+} \rightarrow \mathcal{R}$ is given as

$$
I^{\theta_{1}} \digamma(r)=\frac{1}{\Gamma\left(\theta_{1}\right)} \int_{0}^{r}(r-\tau)^{\theta_{1}-1} \digamma(\tau) d \tau
$$

so that the integral on the right-hand side is pointwise defined on $\mathcal{R}^{+}$.

Lemma 2.3 Let $\theta_{1}>0$ and $\omega_{1} \in \mathcal{C}(0,1) \cap \mathcal{L}^{1}(0,1)$. Then the general solution of $F D E$

$$
D^{\theta_{1}} \omega_{1}(r)=y(r)
$$

is given by

$$
\omega_{1}(r)=I^{\theta_{1}} y(r)+\rho_{0}+\rho_{1} t+\rho_{2} t^{2}+\cdots+\rho_{m-1} t^{m-1},
$$

for some $\rho_{i} \in \mathcal{R}, i=0,1,2, \ldots, m-1$, where $m$ is the smallest integer such that $m \geq \theta_{1}$.

Lemma 2.4 ([37]) Let $\varphi_{p}$ be the p-Laplacian operator.

(i) If $1<p \leq 2, \zeta_{1} \xi_{2}>0$ and $\left|\zeta_{1}\right|,\left|\xi_{2}\right| \geq m>0$, then

$$
\left|\varphi_{p}\left(\zeta_{1}\right)-\varphi_{p}\left(\zeta_{2}\right)\right| \leq(p-1) m^{p-2}\left|\zeta_{1}-\zeta_{2}\right|
$$

(ii) If $p>2$, and $\left|\zeta_{1}\right|,\left|\zeta_{2}\right| \leq M$, then

$$
\left|\varphi_{p}\left(\zeta_{1}\right)-\varphi_{p}\left(\zeta_{2}\right)\right| \leq(p-1) M^{p-2}\left|\zeta_{1}-\zeta_{2}\right|
$$

Definition 2.5 Let $\mathcal{T}: \mathcal{L} \rightarrow \mathcal{L}$. Then the operator equation given by

$$
\mathcal{T} \omega_{1}(r)=\omega_{1}(r), r \in[0,1]
$$

is called Hyers-Ulam stable if, for any $\xi>0$, the inequality

$$
\left\|\omega_{1}-\mathcal{T} \omega_{1}\right\| \leq \xi, \omega_{1} \in[0,1]
$$

has a unique fixed point, say $\omega_{1}{ }^{*}$, with constant $D>0$ such that $\left\|\omega_{1}-\omega_{1}{ }^{*}\right\| \leq D \xi$ holds for all $\omega_{1} \in[0,1]$.

To proceed further, let the following hypothesis hold:

$\left(\mathcal{H}_{1}\right)$ The nonlocal functions $f, g$, where $\omega_{1}, \omega_{2}, x, y \in \mathcal{R}$, satisfy the inequalities:

$$
\begin{aligned}
& \left|f\left(\kappa, \omega_{1}, \omega_{2}\right)-f(\kappa, x, y)\right| \leq \mathcal{K}_{f}\left(\left|\omega_{1}-x\right|+\left|\omega_{2}-y\right|\right), \\
& \left|g\left(\kappa, \omega_{1}, \omega_{2}\right)-g(\kappa, x, y)\right| \leq \mathcal{K}_{g}\left(\left|\omega_{1}-x\right|+\left|\omega_{2}-y\right|\right),
\end{aligned}
$$

where $\mathcal{K}_{f}, \mathcal{K}_{g} \in[0,1)$. 
Theorem 2.6 Let $\gamma \neq \frac{\eta^{\left(\theta_{1}+\alpha-1\right)}}{\Gamma\left(\theta_{1}+\alpha\right)}$. Then for a given $g \in \mathcal{C}([0,1], \mathcal{R})$, the solution of the fractional differential equation

$$
\mathcal{D}^{\theta_{1}^{*}} \varphi_{p}\left(\mathcal{D}^{\theta_{1}} x(r)\right)-g(r)=0, \quad \theta_{1} \in(1,2], \theta_{1}^{*} \in(0,1],
$$

with the boundary condition

$$
\left.\varphi_{p}\left(\mathcal{D}^{\theta_{1}} x(r)\right)\right|_{r=0}=\left.x^{\prime}(r)\right|_{r=0}=0,\left.\quad x(r)\right|_{r=0}=\gamma I^{\alpha-1} h_{1}(\eta)
$$

has a unique solution given by

$$
\begin{aligned}
x(r)= & \frac{1}{\Gamma\left(\theta_{1}\right)} \int_{0}^{r}(r-s)^{\theta_{1}-1} \varphi_{q}\left(I^{\theta_{1}^{*}} g(s)\right) d s \\
& +\frac{\gamma}{\Delta_{1} \Gamma\left(\theta_{1}+\alpha-1\right)} \int_{0}^{\eta}(\eta-s)^{\theta_{1}+\alpha-2} \varphi_{q}\left(I^{\theta_{1}^{*}} g(s)\right) d s .
\end{aligned}
$$

Proof Applying the operator $I^{\theta_{1}^{*}}$ on (2.1) and using Lemma 2.3, we get from (2.1) the following equivalent integral form:

$$
\varphi_{p}\left(\mathcal{D}^{\theta_{1}} x(r)\right)=A_{0}+I^{\theta_{1}^{*}} g(r)
$$

and then, by using condition $\left.\varphi_{p}\left(\mathcal{D}^{\theta_{1}} x(r)\right)\right|_{r=0}=0$, we get $A_{0}=0$. From (2.4), we have

$$
\mathcal{D}^{\theta_{1}} x(r)=\varphi_{q}\left(I^{\theta_{1}^{*}} g(r)\right)
$$

Applying the operator $I_{0}^{\theta_{1}}$ on (2.5) and using Lemma 2.3 again, we get from (2.5) the following equivalent integral form:

$$
x(r)=A_{1}+A_{2} r+I^{\theta_{1}}\left(\varphi_{q} I_{1}^{\theta_{1}^{*}} g(r)\right) .
$$

By using the condition $\left.x^{\prime}(r)\right|_{r=0}=0$ in (2.6), we obtain $A_{2}=0$. Also in view of condition $\left.x(r)\right|_{r=0}=\gamma I^{\alpha-1} x(\eta)$ in (2.6), we get

$$
A_{1}=\frac{\gamma}{\Delta_{1}} I^{\theta_{1}+\alpha-1}\left(\varphi_{q} I^{\theta_{1}^{*}} g(\eta)\right)
$$

where $\Delta_{1}=\left(1-\frac{\gamma}{\Gamma \alpha}\right)$. By substituting the values of $A_{1}$ and $A_{2}$ in (2.6), we get (2.3).

With the help of Theorem 2.6, our coupled FDEs involving the $p$-Laplacian with integral boundary conditions are equivalent to the following Hammerstein-type integral system:

$$
\left\{\begin{aligned}
\omega_{1}(r)= & \left.\frac{1}{\Gamma\left(\theta_{1}\right)} \int_{0}^{r}(r-s)^{\theta_{1}-1} \varphi_{q}\left(I^{\theta_{1}^{*}} f\left(s, \omega_{1}(s), \omega_{2}(s)\right)\right)\right) d s \\
& +\frac{\gamma}{\Delta_{1} \Gamma\left(\theta_{1}+\alpha-1\right)} \int_{0}^{\eta}(\eta-s)^{\theta_{1}+\alpha-2} \varphi_{q}\left(I_{1}^{\theta_{1}^{*}} f\left(s, \omega_{1}(s), \omega_{2}(s)\right)\right) d s, \\
\omega_{2}(r)= & \left.\frac{1}{\Gamma\left(\theta_{2}\right)} \int_{0}^{r}(r-s)^{\theta_{2}-1} \varphi_{q}\left(I_{2}^{\theta_{2}^{*}} g\left(s, \omega_{1}(s), \omega_{2}(s)\right)\right)\right) d s \\
& +\frac{\delta}{\Delta_{2} \Gamma\left(\theta_{2}+\beta-1\right)} \int_{0}^{\xi}(\xi-s)^{\theta_{2}+\beta-2} \varphi_{q}\left(I_{2}^{\theta_{2}^{*}} g\left(s, \omega_{1}(s), \omega_{2}(s)\right)\right) d s .
\end{aligned}\right.
$$




\section{Existence and uniqueness}

For the sake of convenience, we set

$$
\begin{aligned}
& M_{1}=\left(\frac{1}{\Gamma\left(\theta_{1}^{*}+1\right)}\right)^{q-1}\left[\frac{1}{\Gamma\left(\theta_{1}+1\right)}+\frac{\gamma \eta^{\theta_{1}+\alpha-1}}{\Delta_{1} \Gamma\left(\theta_{1}+\alpha\right)}\right], \\
& M_{2}=\left(\frac{1}{\Gamma\left(\theta_{1}^{*}+1\right)}\right)^{q-1}\left[\frac{1}{\Gamma\left(\theta_{2}+1\right)}+\frac{\delta \xi^{\theta_{2}+\beta-1}}{\Delta_{2} \Gamma\left(\theta_{2}+\beta\right)}\right], \\
& M_{0}=\min \left\{1-\left(M_{1} k_{1}+M_{2} \lambda_{1}\right), 1-\left(M_{1} k_{2}+M_{2} \lambda_{2}\right)\right\}, \\
& \boldsymbol{\Delta}_{f}=\frac{(q-1) J_{1}^{q-2}}{\Gamma\left(\theta_{1}^{*}+1\right)}\left[\frac{1}{\Gamma\left(\theta_{1}+1\right)}+\frac{\gamma \eta^{\theta_{1}+\alpha-1}}{\Delta_{1} \Gamma\left(\theta_{1}+\alpha\right)}\right], \\
& \mathbf{\Delta}_{g}=\frac{(q-1) J_{2}^{q-2}}{\Gamma\left(\theta_{1}^{*}+1\right)}\left[\frac{1}{\Gamma\left(\theta_{2}+1\right)}+\frac{\delta \xi^{\theta_{2}+\beta-1}}{\Delta_{2} \Gamma\left(\theta_{2}+\beta\right)}\right] .
\end{aligned}
$$

We define operators $T_{1}, T_{2}: \mathcal{X} \times \mathcal{Y} \rightarrow \mathcal{X} \times \mathcal{Y}$ as

$$
\begin{aligned}
T_{1}\left(\omega_{1}, \omega_{2}\right)(r)= & \left.\frac{1}{\Gamma\left(\theta_{1}\right)} \int_{0}^{r}(r-s)^{\theta_{1}-1} \varphi_{q}\left(I^{\theta_{1}^{*}} f\left(s, \omega_{1}(s), \omega_{2}(s)\right)\right)\right) d s \\
& +\frac{\gamma}{\Delta_{1} \Gamma\left(\theta_{1}+\alpha-1\right)} \int_{0}^{\eta}(\eta-s)^{\theta_{1}+\alpha-2} \varphi_{q}\left(I^{\theta_{1}^{*}} f\left(s, \omega_{1}(s), \omega_{2}(s)\right)\right) d s, \\
T_{2}\left(\omega_{1}, \omega_{2}\right)(r)= & \left.\frac{1}{\Gamma\left(\theta_{2}\right)} \int_{0}^{r}(r-s)^{\theta_{2}-1} \varphi_{q}\left(I^{\theta_{2}^{*}} g\left(s, \omega_{1}(s), \omega_{2}(s)\right)\right)\right) d s \\
& +\frac{\delta}{\Delta_{2} \Gamma\left(\theta_{2}+\beta-1\right)} \int_{0}^{\xi}(\xi-s)^{\theta_{2}+\beta-2} \varphi_{q}\left(I^{\theta_{2}^{*}} g\left(s, \omega_{1}(s), \omega_{2}(s)\right)\right) d s .
\end{aligned}
$$

Lemma $3.1([9,26,30])$ Let $\mathcal{F}: \mathcal{A} \rightarrow \mathcal{A}$ be a completely continuous operator (i.e., a map which, restricted to any bounded set in $\mathcal{A}$, is compact). Let

$$
\varepsilon(\mathcal{F})=\{x \in \mathcal{A}: x=\lambda \mathcal{F}(x), \text { for some } 0<\lambda<1\} .
$$

Then either the set $\varepsilon(F)$ is unbounded, or $\mathcal{F}$ has at least one fixed point.

Theorem 3.2 Suppose that $\gamma \neq \frac{\eta^{\left(\theta_{1}+\alpha-1\right)}}{\Gamma\left(\theta_{1}+\alpha\right)}$ and $\delta \neq \frac{\xi^{\left(\theta_{2}+\beta-1\right)}}{\Gamma\left(\theta_{2}+\beta\right)}$. Assume that there exist real constants $k_{i}, \lambda_{i} \geq 0(i=1,2)$ and $k_{0}>0, \lambda_{0}>0$ such that for all $x_{i} \in \mathcal{R}(i=1,2)$, we have

$$
\begin{aligned}
& \left|f\left(r, x_{1}, x_{2}\right)\right| \leq \varphi_{p}\left(k_{0}+k_{1}\left|x_{1}\right|+k_{2}\left|x_{2}\right|\right), \\
& \left|g\left(r, x_{1}, x_{2}\right)\right| \leq \varphi_{p}\left(\lambda_{0}+\lambda_{1}\left|x_{1}\right|+\lambda_{2}\left|x_{2}\right|\right) .
\end{aligned}
$$

In addition, it is assumed that

$$
M_{1} k_{1}+M_{2} \lambda_{1}<1 \text { and } M_{1} k_{2}+M_{2} \lambda_{2}<1 \text {, }
$$

where $M_{1}$ and $M_{2}$ are given by (3.1) and (3.2) respectively. Then the boundary value problem (1.1) has at least one solution.

Proof First, we show that the operator $T: \mathcal{X} \times \mathcal{Y} \rightarrow \mathcal{X} \times \mathcal{Y}$ is completely continuous. By the continuity of functions $f$ and $g$, the operator $T$ is continuous. Let $\Omega \subset \mathcal{X} \times \mathcal{Y}$ be 
bounded. Then there exist positive constants $L_{1}$ and $L_{2}$ such that

$$
\left|f\left(r, \omega_{1}(r), \omega_{2}(r)\right)\right| \leq \varphi_{p}\left(L_{1}\right), \quad\left|g\left(r, \omega_{1}(r), \omega_{2}(r)\right)\right| \leq \varphi_{p}\left(L_{2}\right), \quad \forall\left(\omega_{1}, \omega_{2}\right) \in \Omega .
$$

Then for any $\left(\omega_{1},, \omega_{2}\right) \in \Omega$, we have

$$
\begin{aligned}
\left|T_{1}\left(\omega_{1}, \omega_{2}\right)(r)\right| & \mid \frac{1}{\Gamma\left(\theta_{1}\right)} \int_{0}^{r}(r-s)^{\theta_{1}-1} \varphi_{q}\left(I_{1}^{\theta_{1}^{*}} f\left(s, \omega_{1}(s), \omega_{2}(s)\right)\right) d s \\
& +\frac{\gamma}{\Delta_{1} \Gamma\left(\theta_{1}+\alpha-1\right)} \int_{0}^{\eta}(\eta-s)^{\theta_{1}+\alpha-2} \varphi_{q}\left(I^{\theta_{1}^{*}} f\left(s, \omega_{1}(s), \omega_{2}(s)\right)\right) d s \mid \\
\leq & \frac{1}{\Gamma\left(\theta_{1}\right)} \int_{0}^{r}(r-s)^{\theta_{1}-1} \varphi_{q}\left(\frac{1}{\Gamma\left(\theta_{1}^{*}\right)} \int_{0}^{r}(r-s)^{\theta_{1}^{*}-1}\left|f\left(s, \omega_{1}(s), \omega_{2}(s)\right)\right| d s\right) \\
& +\frac{\gamma}{\Delta_{1} \Gamma\left(\theta_{1}+\alpha-1\right)} \\
& \times \int_{0}^{\eta}(\eta-s)^{\theta_{1}+\alpha-2} \varphi_{q}\left(\frac{1}{\Gamma\left(\theta_{1}^{*}\right)} \int_{0}^{\eta}(\eta-s)^{\theta_{1}^{*}-1}\left|f\left(s, \omega_{1}(s), \omega_{2}(s)\right)\right|\right) d s \\
\leq & \frac{1}{\Gamma\left(\theta_{1}\right)} \int_{0}^{r}(r-s)^{\theta_{1}-1} \varphi_{q}\left(\frac{1}{\Gamma\left(\theta_{1}^{*}\right)} \int_{0}^{r}(r-s)^{\theta_{1}^{*}-1} \varphi_{p}\left(L_{1}\right) d s\right) \\
& +\frac{\gamma}{\Delta_{1} \Gamma\left(\theta_{1}+\alpha-1\right)} \int_{0}^{\eta}(\eta-s)^{\theta_{1}+\alpha-2} \varphi_{q}\left(\frac{1}{\Gamma\left(\theta_{1}^{*}\right)} \int_{0}^{\eta}(\eta-s)^{\theta_{1}^{*}-1} \varphi_{p}\left(L_{1}\right)\right) d s \\
\leq & L_{1}\left(\frac{1}{\Gamma\left(\theta_{1}^{*}+1\right)}\right)^{q-1}\left[\frac{1}{\Gamma\left(\theta_{1}+1\right)}+\frac{\gamma \eta^{\theta_{1}+\alpha-1}}{\Delta_{1} \Gamma\left(\theta_{1}+\alpha\right)}\right]=L_{1} M_{1} .
\end{aligned}
$$

And also,

$$
\begin{aligned}
&\left|T_{2}\left(\omega_{1}, \omega_{2}\right)(r)\right| \\
&=\mid \frac{1}{\Gamma\left(\theta_{2}\right)} \int_{0}^{r}(r-s)^{\theta_{2}-1} \varphi_{q}\left(I^{\theta_{2}^{*}} g\left(s, \omega_{1}(s), \omega_{2}(s)\right)\right) d s \\
& \quad+\frac{\delta}{\Delta_{2} \Gamma\left(\theta_{2}+\beta-1\right)} \int_{0}^{\xi}(\xi-s)^{\theta_{2}+\beta-2} \varphi_{q}\left(I^{\theta_{2}^{*}} g\left(s, \omega_{1}(s), \omega_{2}(s)\right)\right) d s \mid \\
& \leq \frac{1}{\Gamma\left(\theta_{2}\right)} \int_{0}^{r}(r-s)^{\theta_{2}-1} \varphi_{q}\left(\frac{1}{\Gamma\left(\theta_{2} 2\right)} \int_{0}^{r}(r-s)^{\theta_{2}^{*}-1}\left|g\left(s, \omega_{1}(s), \omega_{2}(s)\right)\right| d s\right) \\
&+\frac{\delta}{\Delta_{2} \Gamma\left(\theta_{2}+\beta-1\right)} \\
& \times \int_{0}^{\xi}(\xi-s)^{\theta_{2}+\beta-2} \varphi_{q}\left(\frac{1}{\Gamma\left(\theta_{2}^{*}\right)} \int_{0}^{\xi}(\xi-s)^{\theta_{2}^{*}-1}\left|g\left(s, \omega_{1}(s), \omega_{2}(s)\right)\right|\right) d s \\
& \leq \frac{1}{\Gamma\left(\theta_{2}\right)} \int_{0}^{r}(r-s)^{\theta_{2}-1} \varphi_{q}\left(\frac{1}{\Gamma\left(\theta_{2}^{*}\right)} \int_{0}^{r}(r-s)^{\theta_{2}^{*}-1} \varphi_{p}\left(L_{2}\right) d s\right) \\
&+\frac{\delta}{\Delta_{2} \Gamma\left(\theta_{2}+\beta-1\right)} \int_{0}^{\xi}(\xi-s)^{\theta_{2}+\beta-2} \varphi_{q}\left(\frac{1}{\Gamma\left(\theta_{2}^{*}\right)} \int_{0}^{\xi}(\xi-s)^{\theta_{2}^{*}-1} \varphi_{p}\left(L_{2}\right)\right) d s \\
& \leq L_{2}\left(\frac{1}{\Gamma\left(\theta_{2}^{*}+1\right)}\right)^{q-1}\left[\frac{1}{\Gamma\left(\theta_{2}+1\right)}+\frac{\delta \xi^{\theta_{2}+\beta-1}}{\Delta_{2} \Gamma\left(\theta_{2}+\beta\right)}\right]=L_{2} M_{2} .
\end{aligned}
$$


Thus, it follows from the above inequalities that the operator $T$ is uniformly bounded. Next we show that $T$ is equicontinuous. Let $0 \leq r_{1} \leq r_{2} \leq 1$. Then we have

$$
\begin{aligned}
& \left|T_{1}\left(\omega_{1}\left(r_{2}\right), \omega_{2}\left(r_{2}\right)\right)-T_{1}\left(\omega_{1}\left(r_{1}\right), \omega_{2}\left(r_{1}\right)\right)\right| \\
& =\mid \frac{1}{\Gamma\left(\theta_{1}\right)} \int_{0}^{r_{2}}\left(r_{2}-s\right)^{\theta_{1}-1} \varphi_{q}\left(\frac{1}{\Gamma\left(\theta_{1}^{*}\right)} \int_{0}^{r}(r-s)^{\theta_{1}^{*}-1} f\left(s, \omega_{1}(s), \omega_{2}(s)\right)\right) d s \\
& -\frac{1}{\Gamma\left(\theta_{1}\right)} \int_{0}^{r_{1}}\left(r_{1}-s\right)^{\theta_{1}-1} \varphi_{q}\left(\frac{1}{\Gamma\left(\theta_{1}^{*}\right)} \int_{0}^{r}(r-s)^{\theta_{1}^{*}-1} f\left(s, \omega_{1}(s), \omega_{2}(s)\right)\right) d s \mid \\
& \leq \mid \frac{1}{\Gamma\left(\theta_{1}\right)} \int_{0}^{r_{2}}\left[\left(r_{2}-s\right)^{\theta_{1}^{*}-1}-\left(r_{1}-s\right)^{\theta_{1}^{*}-1}\right] \\
& \times \varphi_{q}\left(\frac{1}{\Gamma\left(\theta_{1}^{*}\right)} \int_{0}^{r}(r-s)^{\theta_{1}^{*}-1} f\left(s, \omega_{1}(s), \omega_{2}(s)\right)\right) d s \\
& -\frac{1}{\Gamma\left(\theta_{1}\right)} \int_{r_{1}}^{r_{2}}\left(r_{2}-s\right)^{\theta_{1}-1} \varphi_{q}\left(\frac{1}{\Gamma\left(\theta_{1}^{*}\right)} \int_{0}^{r}(r-s)^{\theta_{1}^{*}-1} f\left(s, \omega_{1}(s), \omega_{2}(s)\right)\right) d s \mid \\
& \leq \frac{L_{1}}{\Gamma\left(\theta_{1}+1\right)\left(\Gamma \theta_{1}^{*}+1\right)^{q-1}}\left(r_{1}^{\theta_{1}}-r_{2}^{\theta_{1}}\right), \\
& \left|T_{2}\left(\omega_{1}\left(r_{2}\right), \omega_{2}\left(r_{2}\right)\right)-T_{2}\left(\omega_{1}\left(r_{1}\right), \omega_{2}\left(r_{1}\right)\right)\right| \\
& =\mid \frac{1}{\Gamma\left(\theta_{2}\right)} \int_{0}^{r_{2}}\left(r_{2}-s\right)^{\theta_{2}-1} \varphi_{q}\left(\frac{1}{\Gamma\left(\theta_{2}^{*}\right)} \int_{0}^{r}(r-s)^{\theta_{2}^{*}-1} f\left(s, \omega_{1}(s), \omega_{2}(s)\right)\right) d s \\
& -\frac{1}{\Gamma\left(\theta_{2}\right)} \int_{0}^{r_{1}}\left(r_{1}-s\right)^{\theta_{2}-1} \varphi_{q}\left(\frac{1}{\Gamma\left(\theta_{2}^{*}\right)} \int_{0}^{r}(r-s)^{\theta_{2}^{*}-1} f\left(s, \omega_{1}(s), \omega_{2}(s)\right)\right) d s \mid \\
& \leq \mid \frac{1}{\Gamma\left(\theta_{2}\right)} \int_{0}^{r_{2}}\left[\left(r_{2}-s\right)^{\theta_{2}^{*}-1}-\left(r_{1}-s\right)^{\theta_{2}^{*}-1}\right] \\
& \times \varphi_{q}\left(\frac{1}{\Gamma\left(\theta_{2}^{*}\right)} \int_{0}^{r}(r-s)^{\theta_{2}^{*}-1} f\left(s, \omega_{1}(s), \omega_{2}(s)\right)\right) d s \\
& -\frac{1}{\Gamma\left(\theta_{2}\right)} \int_{r_{1}}^{r_{2}}\left(r_{2}-s\right)^{\theta_{2}-1} \varphi_{q}\left(\frac{1}{\Gamma\left(\theta_{2}^{*}\right)} \int_{0}^{r}(r-s)^{\theta_{2}^{*}-1} f\left(s, \omega_{1}(s), \omega_{2}(s)\right)\right) d s \mid \\
& \leq \frac{L_{1}}{\Gamma\left(\theta_{2}+1\right)\left(\Gamma \theta_{2}^{*}+1\right)^{q-1}}\left(r_{1}^{\theta_{2}}-r_{2}^{\theta_{2}}\right) \text {. }
\end{aligned}
$$

Therefore, the operator $T\left(\omega_{1}, \omega_{2}\right)$ is equicontinuous, and thus the operator $T\left(\omega_{1}, \omega_{2}\right)$ is completely continuous. Finally, it will be verified that the set $\varepsilon=\left\{\left(\omega_{1}, \omega_{2}\right) \in \mathcal{X} \times\right.$ $\left.\mathcal{Y} \mid\left(\omega_{1}, \omega_{2}\right)=\lambda T\left(\omega_{1}, \omega_{2}\right), 0 \leq \lambda \leq 1\right\}$ is bounded. Let $\left(\omega_{1}, \omega_{2}\right) \in \varepsilon$, then $\left(\omega_{1}, \omega_{2}\right)=\lambda T\left(\omega_{1}, \omega_{2}\right)$. For any $r \in[0,1]$, we have

$$
\omega_{1}(r)=\lambda r_{1}\left(\omega_{1}, \omega_{2}\right), \quad \omega_{2}(r)=\lambda T_{2}\left(\omega_{1}, \omega_{2}\right) .
$$

Then

$$
\begin{aligned}
\left|\omega_{1}(r)\right|= & \left(\frac{1}{\Gamma\left(\theta_{1}^{*}+1\right)}\right)^{q-1}\left[\frac{1}{\Gamma\left(\theta_{1}+1\right)}+\frac{\gamma \eta^{\theta_{1}+\alpha-1}}{\Delta_{1} \Gamma\left(\theta_{1}+\alpha\right)}\right] \\
& \times\left(k_{0}+k_{1}\left|\omega_{1}(r)\right|+k_{2}\left|\omega_{2}(r)\right|\right)
\end{aligned}
$$


and

$$
\begin{aligned}
\left|\omega_{2}(r)\right|= & \left(\frac{1}{\Gamma\left(\theta_{2}^{*}+1\right)}\right)^{q-1}\left[\frac{1}{\Gamma\left(\theta_{2}+1\right)}+\frac{\delta \xi^{\theta_{2}+\beta-1}}{\Delta_{2} \Gamma\left(\theta_{2}+\beta\right)}\right] \\
& \times\left(\lambda_{0}+\lambda_{1}\left|\omega_{1}(r)\right|+\lambda_{2}\left|\omega_{2}(r)\right|\right) .
\end{aligned}
$$

Hence we have

$$
\begin{aligned}
& \left\|\omega_{1}\right\|=M_{1}\left(k_{0}+k_{1}\left\|\omega_{1}(r)\right\|+k_{2}\left\|\omega_{2}(r)\right\|\right) \text { and } \\
& \left\|\omega_{2}\right\|=M_{2}\left(\lambda_{0}+\lambda_{1}\left\|\omega_{1}(r)\right\|+\lambda_{2}\left\|\omega_{2}(r)\right\|\right) .
\end{aligned}
$$

From (3.16) we have

$$
\left\|\omega_{1}\right\|+\left\|\omega_{2}\right\|=\left(M_{1} k_{0}+M_{2} \lambda_{0}\right)+\left(M_{1} k_{1}+M_{2} \lambda_{1}\right)\left\|\omega_{1}\right\|+\left(M_{1} k_{2}+M_{2} \lambda_{2}\right)\left\|\omega_{2}\right\| .
$$

Consequently,

$$
\left\|\left(\omega_{1}, \omega_{2}\right)\right\| \leq \frac{M_{1} k_{0}+M_{2} \lambda_{0}}{M_{0}},
$$

for any $r \in[0,1]$, where $M_{0}$ is defined in (3.3), which proves that $\varepsilon$ is bounded. Thus, by Lemma 3.2, operator $T$ has at leat one fixed point. Hence, the boundary value problem (1.1) has at least one solution.

Theorem 3.3 Assume that $f, g:[0,1] \times \mathcal{R}^{2} \rightarrow \mathcal{R}$ are continuous functions and there exist constants $m_{i}, n_{i}, i=1,2$ such that for all $r \in[0,1]$ and $\omega_{1}, \omega_{2}, \hbar_{1}, \hbar_{2} \in \mathcal{R}$,

$$
\begin{aligned}
& \left|f\left(r, \omega_{1}, \hbar_{1}\right)-f\left(r, \omega_{2}, \hbar_{2}\right)\right| \leq m_{1}\left|\omega_{1}-\omega_{2}\right|+n_{1}\left|\hbar_{1}-\hbar_{2}\right| \\
& \left|g\left(r, \omega_{1}, \hbar_{1}\right)-g\left(r, \omega_{2}, \hbar_{2}\right)\right| \leq m_{2}\left|\omega_{1}-\omega_{2}\right|+n_{2}\left|\hbar_{1}-\hbar_{2}\right| .
\end{aligned}
$$

In addition, assume that

$$
\mathbf{\Delta}_{f}\left(m_{1}+m_{2}\right)+\boldsymbol{\Delta}_{g}\left(n_{1}+n_{2}\right)<1
$$

where $\boldsymbol{\Delta}_{f}$ and $\boldsymbol{\Delta}_{g}$ are given by (3.4) and (3.5), respectively. Then the boundary value problem (1.1) has a unique solution.

Proof Consider a bounded set $\left\|T\left(\omega_{1}, \omega_{2}\right)(r)\right\| \leq r$. For $\left(\hbar_{1}, \hbar_{2}\right),\left(\omega_{1}, \omega_{2}\right) \in \mathcal{X} \times \mathcal{Y}$, and for any $r \in[0,1]$, we get

$$
\begin{aligned}
& \left|r_{1}\left(\hbar_{1}, \hbar_{2}\right)(r)-r_{1}\left(\omega_{1}, \omega_{2}\right)(r)\right| \\
& =\mid \frac{1}{\Gamma\left(\theta_{1}\right)} \int_{0}^{r}(r-s)^{\theta_{1}-1} \varphi_{q}\left(\frac{1}{\Gamma\left(\theta_{1}^{*}\right)} \int_{0}^{r}(r-s)^{\theta_{1}^{*}-1} f\left(s, \hbar_{1}(s), \hbar_{2}(s)\right)\right) d s \\
& \quad+\frac{\gamma}{\Delta_{1} \Gamma\left(\theta_{1}+\alpha-1\right)} \int_{0}^{\eta}(\eta-s)^{\theta_{1}+\alpha-2} \varphi_{q}\left(\frac{1}{\Gamma\left(\theta_{1}^{*}\right)} \int_{0}^{r}(r-s)^{\theta_{1}^{*}-1} f\left(s, \hbar_{1}(s), \hbar_{2}(s)\right)\right) d s \\
& \quad-\frac{1}{\Gamma\left(\theta_{1}\right)} \int_{0}^{r}(r-s)^{\theta_{1}-1} \varphi_{q}\left(\frac{1}{\Gamma\left(\theta_{1}^{*}\right)} \int_{0}^{r}(r-s)^{\theta_{1}^{*}-1} f\left(s, \omega_{1}(s), \omega_{2}(s)\right)\right) d s
\end{aligned}
$$




$$
\begin{aligned}
& -\frac{\gamma}{\Delta_{1} \Gamma\left(\theta_{1}+\alpha-1\right)} \int_{0}^{\eta}(\eta-s)^{\theta_{1}+\alpha-2} \varphi_{q}\left(\frac{1}{\Gamma\left(\theta_{1}^{*}\right)} \int_{0}^{r}(r-s)^{\theta_{1}^{*}-1} f\left(s, \omega_{1}(s), \omega_{2}(s)\right)\right) d s \mid \\
\leq & \frac{(q-1) J_{1}^{q-2}}{\Gamma\left(\theta_{1}\right)} \int_{0}^{r}\left|(r-s)^{\theta_{1}-1}\right| \frac{1}{\Gamma\left(\theta_{1}^{*}\right)} \int_{0}^{r}\left|(r-s)^{\theta_{1}^{*}-1}\right| \mid f\left(s, \hbar_{1}(s), \hbar_{2}(s)\right) \\
& -f\left(s, \omega_{1}(s), \omega_{2}(s)\right) \mid d s \\
& +\frac{(q-1) J_{1}^{q-2} \gamma}{\Delta_{1} \Gamma\left(\theta_{1}+\alpha-1\right)} \int_{0}^{\eta}\left|(\eta-s)^{\theta_{1}+\alpha-2}\right| \frac{1}{\Gamma\left(\theta_{1}^{*}\right)} \int_{0}^{r}\left|(r-s)^{\theta_{1}^{*}-1}\right| \mid f\left(s, \hbar_{1}(s), \hbar_{2}(s)\right) \\
& -f\left(s, \omega_{1}(s), \omega_{2}(s)\right) \mid d s \\
\leq & \frac{(q-1) J_{1}^{q-2}}{\Gamma\left(\theta_{1}^{*}+1\right)}\left[\frac{1}{\Gamma\left(\theta_{1}+1\right)}+\frac{\gamma \eta^{\theta_{1}+\alpha-1}}{\Delta_{1} \Gamma\left(\theta_{1}+\alpha\right)}\right]\left(m_{1}\left|\hbar_{1}-\omega_{1}\right|+m_{2}\left|\hbar_{2}-\omega_{2}\right|\right) \\
\leq & \mathbf{\Delta}_{f}\left(m_{1}+m_{2}\right)\left(\left|\hbar_{1}-\omega_{1}\right|+\left|\hbar_{2}-\omega_{2}\right|\right) .
\end{aligned}
$$

Similarly, we have

$$
\begin{aligned}
&\left|T_{2}\left(\hbar_{1}, \hbar_{2}\right)(r)-T_{2}\left(\omega_{1}, \omega_{2}\right)(r)\right| \\
&=\mid \frac{1}{\Gamma\left(\theta_{2}\right)} \int_{0}^{r}(r-s)^{\theta_{2}-1} \varphi_{q}\left(\frac{1}{\Gamma\left(\theta_{2}^{*}\right)} \int_{0}^{r}(r-s)^{\theta_{2}^{*}-1} g\left(s, \hbar_{1}(s), \hbar_{2}(s)\right)\right) d s \\
&+\frac{\delta}{\Delta_{2} \Gamma\left(\theta_{2}+\beta-1\right)} \int_{0}^{\xi}(\xi-s)^{\theta_{2}+\beta-2} \varphi_{q}\left(\frac{1}{\Gamma\left(\theta_{2}^{*}\right)} \int_{0}^{r}(r-s)^{\theta_{2}^{*}-1} g\left(s, \hbar_{1}(s), \hbar_{2}(s)\right)\right) d s \\
&-\frac{1}{\Gamma\left(\theta_{2}\right)} \int_{0}^{r}(r-s)^{\theta_{2}-1} \varphi_{q}\left(\frac{1}{\Gamma\left(\theta_{2}^{*}\right)} \int_{0}^{r}(r-s)^{\theta_{2}^{*}-1} g\left(s, \omega_{1}(s), \omega_{2}(s)\right)\right) d s \\
&-\frac{\delta}{\Delta_{2} \Gamma\left(\theta_{2}+\beta-1\right)} \int_{0}^{\xi}(\xi-s)^{\theta_{2}+\beta-2} \varphi_{q}\left(\frac{1}{\Gamma\left(\theta_{2}^{*}\right)} \int_{0}^{r}(r-s)^{\theta_{2}^{*}-1} g\left(s, \omega_{1}(s), \omega_{2}(s)\right)\right) d s \\
&-\frac{1}{\Gamma\left(\theta_{2}\right)} \int_{0}^{r}(r-s)^{\theta_{2}-1} \varphi_{q}\left(\frac{1}{\Gamma\left(\theta_{2}^{*}\right)} \int_{0}^{r}(r-s)^{\theta_{2}^{*}-1} g\left(s, \omega_{1}(s), \omega_{2}(s)\right)\right) d s \\
&-\frac{\delta}{\Delta_{2} \Gamma\left(\theta_{2}+\beta-1\right)} \int_{0}^{\xi}(\xi-s)^{\theta_{2}+\beta-2} \varphi_{q}\left(\frac{1}{\Gamma\left(\theta_{2}^{*}\right)} \int_{0}^{r}(r-s)^{\theta_{2}^{*}-1} g\left(s, \omega_{1}(s), \omega_{2}(s)\right)\right) d s \mid \\
& \leq \frac{(q-1) J_{2}^{q-2}}{\Gamma\left(\theta_{2}\right)} \int_{0}^{r}\left|(r-s)^{\theta_{2}-1}\right| \frac{1}{\Gamma\left(\theta_{2}^{*}\right)} \int_{0}^{r}\left|(r-s)^{\theta_{2}^{*}-1}\right| \mid g\left(s, \hbar_{1}(s), \hbar_{2}(s)\right) \\
&-g\left(s, \omega_{1}(s), \omega_{2}(s)\right) \mid d s \\
&+\frac{(q-1) J_{2}^{q-2} \delta}{\Delta_{2} \Gamma\left(\theta_{2}+\beta-1\right)} \int_{0}^{\xi}\left|(\xi-s)^{\theta_{2}+\beta-2}\right| \frac{1}{\Gamma\left(\theta_{2}^{*}\right)} \int_{0}^{r}\left|(r-s)^{\theta_{2}^{*}-1}\right| \mid g\left(s, \hbar_{1}(s), \hbar_{2}(s)\right) \\
&-g\left(s, \omega_{1}(s), \omega_{2}(s)\right) \mid d s \\
& \leq \frac{(q-1) J_{2}^{(q-2)}}{\Gamma\left(\theta_{2}^{*}+1\right)}\left[\frac{1}{\Gamma\left(\theta_{2}+1\right)}+\frac{\delta \xi^{\theta_{2}+\beta-1}}{\Delta_{2} \Gamma\left(\theta_{2}+\beta\right)}\right]\left(n_{1}\left|\hbar_{1}-\omega_{1}\right|+n_{2}\left|\hbar_{2}-\omega_{2}\right|\right) \\
& \leq \mathbf{\Delta}_{g}\left(n_{1}+n_{2}\right)\left(\left\|\hbar_{1}-\omega_{1}\right\|+\left\|\hbar_{2}-\omega_{2}\right\|\right) . \\
&
\end{aligned}
$$

Therefore, by (3.20) and (3.21), we have

$$
\begin{aligned}
& \left\|T\left(\hbar_{1}, \hbar_{2}\right)(r)-T\left(\omega_{1}, \omega_{2}\right)(r)\right\| \\
& \quad \leq\left[\mathbf{\Lambda}_{f}\left(m_{1}+m_{2}\right)+\mathbf{\Delta}_{g}\left(n_{1}+n_{2}\right)\right]\left(\left\|\hbar_{1}-\omega_{1}\right\|+\left\|\hbar_{2}-\omega_{2}\right\|\right) .
\end{aligned}
$$


Hence $\boldsymbol{\Delta}_{f}\left(m_{1}+m_{2}\right)+\boldsymbol{\Delta}_{g}\left(n_{1}+n_{2}\right)<1$, and therefore $T$ is a contraction operator. So by Banach's fixed point theorem, the operator $T$ has a unique fixed point, which is the unique solution of problem (1.1).

\section{Hyers-Ulam stability of the coupled system}

Definition 4.1 The coupled system of Hammerstein-type integral equations (2.8) is Hyres-Ulam stable if there exist positive constants $D_{i}>0(i=1,2)$ satisfying:

For $\varrho_{i}>0, i=1,2$, if

$$
\begin{aligned}
& \mid \omega_{1}(r)-\frac{1}{\Gamma\left(\theta_{1}\right)} \int_{0}^{r}(r-s)^{\theta_{1}-1} \varphi_{q}\left(I^{\theta_{1}^{*}} f\left(s, \omega_{1}(s), \omega_{2}(s)\right)\right) d s \\
& \quad-\frac{\gamma}{\Delta_{1} \Gamma\left(\theta_{1}+\alpha-1\right)} \int_{0}^{\eta}(\eta-s)^{\theta_{1}+\alpha-2} \varphi_{q}\left(I^{\theta_{1}^{*}} f\left(s, \omega_{1}(s), \omega_{2}(s)\right)\right) d s \mid \leq \varrho_{1}, \\
& \mid \omega_{2}(r)-\frac{1}{\Gamma\left(\theta_{2}\right)} \int_{0}^{r}(r-s)^{\theta_{2}-1} \varphi_{q}\left(I^{\theta_{2}^{*}} g\left(s, \omega_{1}(s), \omega_{2}(s)\right)\right) d s \\
& \quad-\frac{\delta}{\Delta_{2} \Gamma\left(\theta_{2}+\beta-1\right)} \int_{0}^{\xi}(\xi-s)^{\theta_{2}+\beta-2} \varphi_{q}\left(I^{\theta_{2}^{*}} g\left(s, \omega_{1}(s), \omega_{2}(s)\right)\right) d s \mid \leq \varrho_{2},
\end{aligned}
$$

there exist $\left(\omega_{1}^{*}(r), \omega_{2}^{*}(r)\right)$, satisfying

$$
\begin{aligned}
\omega_{1}^{*}(r)= & \frac{1}{\Gamma\left(\theta_{1}\right)} \int_{0}^{r}(r-s)^{\theta_{1}-1} \varphi_{q}\left(I^{\theta_{1}^{*}} f\left(s, \omega_{1}^{*}(s), \omega_{2}^{*}(s)\right)\right) d s \\
& +\frac{\gamma}{\Delta_{1} \Gamma\left(\theta_{1}+\alpha-1\right)} \int_{0}^{\eta}(\eta-s)^{\theta_{1}+\alpha-2} \varphi_{q}\left(I^{\theta_{1}^{*}} f\left(s, \omega_{1}^{*}(s), \omega_{2}^{*}(s)\right)\right) d s, \\
\omega_{2}^{*}(r)= & \frac{1}{\Gamma\left(\theta_{2}\right)} \int_{0}^{r}(r-s)^{\theta_{2}-1} \varphi_{q}\left(I^{\theta_{2}^{*}} g\left(s, \omega_{1}^{*}(s), \omega_{2}^{*}(s)\right)\right) d s \\
& +\frac{\delta}{\Delta_{2} \Gamma\left(\theta_{2}+\beta-1\right)} \int_{0}^{\xi}(\xi-s)^{\theta_{2}+\beta-2} \varphi_{q}\left(I^{\theta_{2}^{*}} g\left(s, \omega_{1}^{*}(s), \omega_{2}^{*}(s)\right)\right) d s,
\end{aligned}
$$

such that

$$
\begin{aligned}
& \left|\omega_{1}(r)-\omega_{1}^{*}(r)\right| \leq D_{1} \varrho_{1}, x \in[0,1], \\
& \left|\omega_{2}(r)-\omega_{2}^{*}(r)\right| \leq D_{2} \varrho_{2}, x \in[0,1] .
\end{aligned}
$$

In the present section, we derive the Hyers-Ulam type stability for the solution of the considered problem.

Theorem 4.2 By the assumption that $f, g:[0,1] \times \mathcal{R}^{2} \rightarrow \mathcal{R}$ are continuous functions and there exist constants $m_{i}, n_{i}, i=1,2$ such that for all $r \in[0,1]$ and $\omega_{1}, \omega_{2}, \hbar_{1}, \hbar_{2} \in \mathcal{R}$,

$$
\begin{aligned}
& \left|f\left(r, \omega_{1}, \hbar_{1}\right)-f\left(r, \omega_{2}, \hbar_{2}\right)\right| \leq m_{1}\left|\omega_{1}-\omega_{2}\right|+n_{1}\left|\hbar_{1}-\hbar_{2}\right|, \\
& \left|g\left(r, \omega_{1}, \hbar_{1}\right)-g\left(r, \omega_{2}, \hbar_{2}\right)\right| \leq m_{2}\left|\omega_{1}-\omega_{2}\right|+n_{2}\left|\hbar_{1}-\hbar_{2}\right|,
\end{aligned}
$$


Proof By Theorem 3.3 and Definition 4.1, let $\left(\omega_{1}(r), \omega_{2}(r)\right)$ be the exact solution, and $\left(\omega_{1}^{*}(r), \omega_{2}^{*}(r)\right)$ be any other solution of system (2.8). Then, with the help of (2.8), we have

$$
\begin{aligned}
& \left|\omega_{1}(r)-\omega_{1}^{*}(r)\right| \leq \mid \frac{1}{\Gamma\left(\theta_{1}\right)} \int_{0}^{r}(r-s)^{\theta_{1}-1} \varphi_{q}\left(\frac{1}{\Gamma\left(\theta_{1}^{*}\right)} \int_{0}^{r}(r-s)^{\theta_{1}^{*}-1} f\left(s, \omega_{1}(s), \omega_{2}(s)\right)\right) d s \\
& +\frac{\gamma}{\Delta_{1} \Gamma\left(\theta_{1}+\alpha-1\right)} \int_{0}^{\eta}(\eta-s)^{\theta_{1}+\alpha-2} \\
& \times \varphi_{q}\left(\frac{1}{\Gamma\left(\theta_{1}^{*}\right)} \int_{0}^{r}(r-s)^{\theta_{1}^{*}-1} f\left(s, \omega_{1}(s), \omega_{2}(s)\right)\right) d s \\
& -\frac{1}{\Gamma\left(\theta_{1}\right)} \int_{0}^{r}(r-s)^{\theta_{1}-1} \varphi_{q}\left(\frac{1}{\Gamma\left(\theta_{1}^{*}\right)} \int_{0}^{r}(r-s)^{\theta_{1}^{*}-1} f\left(s, \omega_{1}^{*}(s), \omega_{2}^{*}(s)\right)\right) d s \\
& -\frac{\gamma}{\Delta_{1} \Gamma\left(\theta_{1}+\alpha-1\right)} \int_{0}^{\eta}(\eta-s)^{\theta_{1}+\alpha-2} \\
& \times \varphi_{q}\left(\frac{1}{\Gamma\left(\theta_{1}^{*}\right)} \int_{0}^{r}(r-s)^{\theta_{1}^{*}-1} f\left(s, \omega_{1}^{*}(s), \omega_{2}^{*}(s)\right)\right) d s \mid \\
& \leq \frac{1}{\Gamma\left(\theta_{1}\right)} \int_{0}^{r}\left|(r-s)^{\theta_{1}-1}\right| \mid \varphi_{q}\left(\frac{1}{\Gamma\left(\theta_{1}^{*}\right)} \int_{0}^{r}(r-s)^{\theta_{1}^{*}-1} g\left(s, \omega_{1}(s), \omega_{2}(s)\right) d s\right) \\
& -\varphi_{q}\left(\frac{1}{\Gamma\left(\theta_{1}^{*}\right)} \int_{0}^{r}(r-s)^{\theta_{1}^{*}-1} g\left(s, \omega_{1}^{*}(s), \omega_{2}^{*}(s)\right) d s\right) \\
& +\frac{\gamma}{\Delta_{1} \Gamma\left(\theta_{1}+\alpha-1\right)} \int_{0}^{\eta}\left|(\eta-s)^{\theta_{1}+\alpha-2}\right| \\
& \times \mid \varphi_{q}\left(\frac{1}{\Gamma\left(\theta_{1}^{*}\right)} \int_{0}^{r}(r-s)^{\theta_{1}^{*}-1} g\left(s, \omega_{1}(s), \omega_{2}(s)\right) d s\right) \\
& -\varphi_{q}\left(\frac{1}{\Gamma\left(\theta_{1}^{*}\right)} \int_{0}^{r}(r-s)^{\theta_{1}^{*}-1} g\left(s, \omega_{1}^{*}(s), \omega_{2}^{*}(s)\right) d s\right) \\
& \leq \frac{(q-1) J_{1}^{q-2}}{\Gamma\left(\theta_{1}\right)} \int_{0}^{r}\left|(r-s)^{\theta_{1}-1}\right| \frac{1}{\Gamma\left(\theta_{1}^{*}\right)} \int_{0}^{r}\left|(r-s)^{\theta_{1}^{*}-1}\right| \mid f\left(s, \omega_{1}(s), \omega_{2}(s)\right) \\
& -f\left(s, \omega_{1}^{*}(s), \omega_{2}^{*}(s)\right) \mid d s \\
& +\frac{(q-1) J_{1}^{q-2} \gamma}{\Delta_{1} \Gamma\left(\theta_{1}+\alpha-1\right)} \int_{0}^{\eta}\left|(\eta-s)^{\theta_{1}+\alpha-2}\right| \\
& \times \frac{1}{\Gamma\left(\theta_{1}^{*}\right)} \int_{0}^{r}\left|(r-s)^{\theta_{1}^{*}-1}\right|\left|f\left(s, \omega_{1}(s), \omega_{2}(s)\right)-f\left(s, \omega_{1}^{*}(s), \omega_{2}^{*}(s)\right)\right| d s \\
& \leq \frac{(q-1) J_{1}^{q-2} \mathcal{K}_{f}}{\Gamma\left(\theta_{1}+1\right) \Gamma\left(\theta_{1}^{*}+1\right)}\left(\left|\omega_{1}(r)-\omega_{1}^{*}(r)\right|+\left|\omega_{2}(r)-\omega_{2}^{*}(r)\right|\right) \\
& +\frac{(q-1) J_{1}^{q-2} \gamma \eta^{\theta_{1}+\alpha-1} \mathcal{K}_{f}}{\Delta_{1} \Gamma\left(\theta_{1}+\alpha\right) \Gamma\left(\theta_{1}^{*}+1\right)}\left(\left|\omega_{1}(r)-\omega_{1}^{*}(r)\right|+\left|\omega_{2}(r)-\omega_{2}^{*}(r)\right|\right),
\end{aligned}
$$

which implies that

$$
\begin{aligned}
\left\|\omega_{1}-\omega_{1}^{*}\right\| \leq & \frac{(q-1) J_{1}^{q-2} \mathcal{K}_{f}}{\Gamma\left(\theta_{1}^{*}+1\right)}\left[\frac{1}{\Gamma\left(\theta_{1}+1\right)}+\frac{\gamma \eta^{\theta_{1}+\alpha-1}}{\Delta_{1} \Gamma\left(\theta_{1}+\alpha\right)}\right] \\
& \times\left(\left\|\omega_{1}-\omega_{1}^{*}\right\|+\left\|\omega_{2}-\omega_{2}^{*}\right\|\right) \\
\leq & D_{1} \varrho_{1}
\end{aligned}
$$


where $D_{1}=\frac{(q-1))_{1}^{q-2} \mathcal{K}_{f}}{\Gamma\left(\theta_{1}^{*}+1\right)}\left[\frac{1}{\Gamma\left(\theta_{1}+1\right)}+\frac{\gamma \eta^{\theta_{1}+\alpha-1}}{\Delta_{1} \Gamma\left(\theta_{1}+\alpha\right)}\right]$. Similarly, we further have

$$
\begin{aligned}
& \left|\omega_{2}(r)-\omega_{2}^{*}(r)\right| \leq \mid \frac{1}{\Gamma\left(\theta_{2}\right)} \int_{0}^{r}(r-s)^{\theta_{2}-1} \varphi_{q}\left(\frac{1}{\Gamma\left(\theta_{2}^{*}\right)} \int_{0}^{r}(r-s)^{\theta_{2}^{*}-1} g\left(s, \omega_{1}(s), \omega_{2}(s)\right)\right) d s \\
& +\frac{\delta}{\Delta_{2} \Gamma\left(\theta_{2}+\beta-1\right)} \\
& \times \int_{0}^{\xi}(\xi-s)^{\theta_{2}+\beta-2} \varphi_{q}\left(\frac{1}{\Gamma\left(\theta_{2}^{*}\right)} \int_{0}^{r}(r-s)^{\theta_{2}^{*}-1} g\left(s, \omega_{1}(s), \omega_{2}(s)\right)\right) d s \\
& -\frac{1}{\Gamma\left(\theta_{2}\right)} \int_{0}^{r}(r-s)^{\theta_{2}-1} \varphi_{q}\left(\frac{1}{\Gamma\left(\theta_{2}^{*}\right)} \int_{0}^{r}(r-s)^{\theta_{2}^{*}-1} g\left(s, \omega_{1}^{*}(s), \omega_{2}^{*}(s)\right)\right) d s \\
& -\frac{\delta}{\Delta_{2} \Gamma\left(\theta_{2}+\beta-1\right)} \int_{0}^{\xi}(\xi-s)^{\theta_{2}+\beta-2} \\
& \times \varphi_{q}\left(\frac{1}{\Gamma\left(\theta_{2}^{*}\right)} \int_{0}^{r}(r-s)^{\theta_{2}^{*}-1} g\left(s, \omega_{1}^{*}(s), \omega_{2}^{*}(s)\right)\right) d s \mid \\
& \leq \frac{1}{\Gamma\left(\theta_{2}\right)} \int_{0}^{r}\left|(r-s)^{\theta_{2}-1}\right| \mid \varphi_{q}\left(\frac{1}{\Gamma\left(\theta_{2}^{*}\right)} \int_{0}^{r}(r-s)^{\theta_{2}^{*}-1} g\left(s, \omega_{1}(s), \omega_{2}(s)\right) d s\right) \\
& -\varphi_{q}\left(\frac{1}{\Gamma\left(\theta_{2}^{*}\right)} \int_{0}^{r}(r-s)^{\theta_{2}^{*}-1} g\left(s, \omega_{1}^{*}(s), \omega_{2}^{*}(s)\right) d s\right) \\
& +\frac{\delta}{\Delta_{2} \Gamma\left(\theta_{2}+\beta-1\right)} \\
& \times \int_{0}^{\xi}\left|(\xi-s)^{\theta_{2}+\beta-2}\right| \mid \varphi_{q}\left(\frac{1}{\Gamma\left(\theta_{2}^{*}\right)} \int_{0}^{r}(r-s)^{\theta_{2}^{*}-1} g\left(s, \omega_{1}(s), \omega_{2}(s)\right) d s\right) \\
& -\varphi_{q}\left(\frac{1}{\Gamma\left(\theta_{2}^{*}\right)} \int_{0}^{r}(r-s)^{\theta_{2}^{*}-1} g\left(s, \omega_{1}^{*}(s), \omega_{2}^{*}(s)\right) d s\right) \\
& \leq \frac{(q-1) J_{2}^{q-2}}{\Gamma\left(\theta_{2}\right)} \int_{0}^{r}\left|(r-s)^{\theta_{2}-1}\right| \frac{1}{\Gamma\left(\theta_{2}^{*}\right)} \int_{0}^{r}\left|(r-s)^{\theta_{2}^{*}-1}\right| \mid g\left(s, \omega_{1}(s), \omega_{2}(s)\right) \\
& -g\left(s, \omega_{1}^{*}(s), \omega_{2}^{*}(s)\right) \mid d s \\
& +\frac{(q-1) J_{2}^{q-2} \delta}{\Delta_{2} \Gamma\left(\theta_{2}+\beta-1\right)} \int_{0}^{\xi}\left|(\xi-s)^{\theta_{2}+\beta-2}\right| \frac{1}{\Gamma\left(\theta_{2}^{*}\right)} \\
& \times \int_{0}^{r}\left|(r-s)^{\theta_{2}^{*}-1}\right|\left|g\left(s, \omega_{1}(s), \omega_{2}(s)\right)-g\left(s, \omega_{1}^{*}(s), \omega_{2}^{*}(s)\right)\right| d s \\
& \leq \frac{(q-1) J_{2}^{q-2} \mathcal{K}_{g}}{\Gamma\left(\theta_{2}+1\right) \Gamma\left(\theta_{2}^{*}+1\right)}\left(\left|\omega_{1}(r)-\omega_{1}^{*}(r)\right|+\left|\omega_{2}(r)-\omega_{2}^{*}(r)\right|\right) \\
& +\frac{(q-1) J_{2}^{q-2} \delta \xi^{\theta_{2}+\alpha-1} \mathcal{K}_{g}}{\Delta_{2} \Gamma\left(\theta_{2}+\beta\right) \Gamma\left(\theta_{2}^{*}+1\right)}\left(\left|\omega_{1}(r)-\omega_{1}^{*}(r)\right|+\left|\omega_{2}(r)-\omega_{2}^{*}(r)\right|\right),
\end{aligned}
$$

which implies that

$$
\begin{aligned}
\left\|\omega_{2}-\omega_{2}^{*}\right\| \leq & \frac{(q-1) J_{2}^{q-2} \mathcal{K}_{g}}{\Gamma\left(\theta_{2}^{*}+1\right)}\left[\frac{1}{\Gamma\left(\theta_{2}+1\right)}+\frac{\delta \xi^{\theta_{2}+\beta-1}}{\Delta_{2} \Gamma\left(\theta_{2}+\beta\right)}\right] \\
& \times\left(\left\|\omega_{1}-\omega_{1}^{*}\right\|+\left\|\omega_{2}-\omega_{2}^{*}\right\|\right) \\
\leq & D_{2} \varrho_{2},
\end{aligned}
$$


where $D_{2}=\frac{(q-1) J_{2}^{q-2} \mathcal{K}_{g}}{\Gamma\left(\theta_{2}^{*}+1\right)}\left[\frac{1}{\Gamma\left(\theta_{2}+1\right)}+\frac{\delta \xi^{\theta_{2}+\beta-1}}{\Delta_{2} \Gamma\left(\theta_{2}+\beta\right)}\right]$. Hence in view of (4.5) and (4.7), the system of integral equations (2.8) is Hyers-Ulam stable, and consequently, the solution of system (1.1) is Hyers-Ulam stable.

\section{Illustrative example}

Example 5.1 Consider the following coupled FDEs involving the nonlinear $p$-Laplacian operator with IBCs:

$$
\begin{aligned}
& \mathcal{D}^{\frac{1}{2}} \varphi_{3}\left(\mathcal{D}^{\frac{3}{2}} \omega_{1}(r)\right)=\frac{e^{-2 r} \omega_{1}(r)}{100}+\frac{\sin \left|\omega_{1}(r)\right|+\cos \left|\omega_{2}(r)\right|}{2\left(25+r^{2}\right)}, \quad r \in[0,1], \\
& \mathcal{D}^{\frac{1}{2}} \varphi_{3}\left(\mathcal{D}^{\frac{3}{2}} \omega_{2}(r)\right)=\frac{\sin \left|\omega_{1}(r)\right|+\left|\omega_{2}(r)\right|}{10(r+1)}+\frac{e^{-2 r} \omega_{2}(r)}{20}, \quad r \in[0,1], \\
& \left.\varphi_{3}\left(\mathcal{D}^{\frac{3}{2}} \omega_{1}(r)\right)\right|_{r=0}=\left.\omega_{1}^{\prime}(r)\right|_{r=0}=0,\left.\quad \omega_{1}(r)\right|_{r=0}=\sqrt{2} I^{\frac{1}{3}} \omega_{1}\left(\frac{1}{2}\right), \\
& \left.\varphi_{3}\left(\mathcal{D}^{\frac{3}{2}} \omega_{2}(r)\right)\right|_{r=0}=\left.v^{\prime}(r)\right|_{r=0}=0,\left.\quad \omega_{2}(r)\right|_{r=0}=\sqrt{3} I^{\frac{1}{2}} \omega_{2}\left(\frac{1}{3}\right) .
\end{aligned}
$$

The suggested parameter values are $\theta_{i}=3 / 2, \theta_{i}^{*}=1 / 2(i=1,2), p=3, \gamma=\sqrt{2}, \delta=\sqrt{3}$, $\eta=1 / 2, \xi=1 / 3, p=4 / 3, q=3 / 2, f\left(r, \omega_{1}, \omega_{2}\right)=\frac{e^{-3 r} \omega_{1}(r)}{30}+\frac{\sin \left|\omega_{1}(r)\right|+\cos \left|\omega_{2}(r)\right|}{\left(50+r^{2}\right)}$ and $g\left(r, \omega_{1}, \omega_{2}\right)=$ $\frac{r^{3}+\sin \left|\omega_{1}(r)\right|+\left|\omega_{2}(r)\right|}{25}+\frac{e^{-2 r} \omega_{1}(r)}{20}$. Further, we have

$$
\begin{aligned}
\left|f\left(r, \omega_{1}, \omega_{2}\right)-f\left(r, \omega_{1}, \omega_{2}\right)\right| & \leq \frac{1}{50}\left|\omega_{1}-\omega_{2}\right|+\frac{1}{50}\left|\omega_{1}-\omega_{2}\right|, \\
\left|g\left(r, \omega_{1}, \omega_{2}\right)-g\left(r, \omega_{1}, \omega_{2}\right)\right| & \leq \frac{1}{10}\left|\omega_{1}-\omega_{2}\right|+\frac{1}{10}\left|\omega_{1}-\omega_{2}\right| .
\end{aligned}
$$

Therefore, we have $m_{1}=0.3=m_{2}, n_{1}=0.2=n_{2}$

$$
\begin{aligned}
\mathbf{\Delta}_{f}\left(m_{1}+m_{2}\right)+\mathbf{\Delta}_{g}\left(n_{1}+n_{2}\right)= & \frac{\left(\frac{3}{2}-1\right) 2^{\frac{-1}{2}}}{\Gamma \frac{3}{2}}\left[\frac{1}{\Gamma \frac{5}{2}}+\frac{\sqrt{2}\left(\frac{1}{2}\right)^{\frac{11}{6}}}{\left(1-\frac{\sqrt{2}}{\Gamma^{\frac{4}{3}}}\right) \Gamma \frac{17}{6}}\right](0.3+0.3) \\
& +\frac{\left(\frac{3}{2}-1\right) 2^{\frac{-1}{2}}}{\Gamma \frac{3}{2}}\left[\frac{1}{\Gamma \frac{5}{2}}+\frac{\sqrt{3}\left(\frac{1}{3}\right)^{\frac{11}{6}}}{\left(1-\frac{\sqrt{3}}{\Gamma^{\frac{4}{3}}}\right) \Gamma \frac{17}{6}}\right](0.2+0.2)
\end{aligned}
$$

$<1$.

Hence all the conditions of Theorem 3.3 are satisfied, and so the coupled system (5.1) has a unique solution and is Hyres-Ulam stable.

\section{Conclusion}

In this paper we investigated existence and uniqueness of solutions for coupled fractional differential equations involving the nonlinear $p$-Laplacian operator with integral boundary conditions, by using nonlinear Leray-Schauder-type alternative and Banach's fixed point theorem. We have also developed some conditions to prove Hyres-Ulam stability. An illustrative example was provided to demonstrate the results. For further studies, we suggest investigating our problem (1.1) for multiplicity results and exponential stability. Readers may also consider the problem for the new established derivative known as ABC fractional derivative. 


\section{Acknowledgements}

The authors are thankful to the anonymous reviewers and the editorial board for their help in the improvement of the quality of our paper. The work was supported by National Natural Science Foundation of China (Nos. 11572111, 11772121).

\section{Funding}

The work was supported by National Natural Science Foundation of China (Nos. 11572111, 11772121).

\section{Competing interests}

The authors have no competing interests regarding the publication of this article.

\section{Authors' contributions}

All the authors have equal contributions in this article. All authors read and approved the final manuscript.

\section{Author details}

${ }^{1}$ College of Engineering Mechanics and Materials, Hohai University, Nanjing, P.R. China. ${ }^{2}$ Shaheed Benazir Bhutto University, Dir Upper, Pakistan. ${ }^{3}$ University of Peshawar, Peshawar, Pakistan. ${ }^{4}$ Department of Mathematical Sciences, United Arab Emirates University, Abu Dhabi, UAE.

\section{Publisher's Note}

Springer Nature remains neutral with regard to jurisdictional claims in published maps and institutional affiliations.

Received: 11 May 2018 Accepted: 19 November 2018 Published online: 07 December 2018

\section{References}

1. Laskin, N.: Fractional market dynamics. Physica A 287, 482-492 (2000)

2. Laskin, N.: Fractional quantum mechanics and Levy path integrals. Phys. Lett. A 298, 298-305 (2000)

3. Pierri, M., O'Regan, D., Rolnik, V.: Existence of solutions for semi-linear abstract differential equations with not instantaneous impulses. Appl. Math. Comput. 219, 6743-6749 (2013)

4. McCluskey, C.C.: A model of HIV/AIDS with staged progression and amelioration. Math. Biosci. 181, 1-6 (2003)

5. Biçer, E., Tunç, C.: New theorems for Hyers-Ulam stability of Lienard equation with variable time lags. Int. J. Math. Comput. Sci. 3, 231-242 (2018)

6. Ran-Chao, W., Xin-Dong, H., Li-Ping, C.: Finite-time stability of fractional-order neural networks with delay. Commun Theor. Phys. 60(2), 189 (2013)

7. Hyers, D.: On the stability of the linear functional equation. Proc. Natl. Acad. Sci. USA 27, 222-224 (1941)

8. Laskin, N.: Fractional quantum mechanics. Phys. Rev. E 62(3), 3135 (2000)

9. Shah, K., Tunc, C.: Existence theory and stability analysis to a system of boundary value problem. J. Taibah Univ. Sci. 11, 1330-1342 (2017)

10. Jarad, F., Abdeljawad, T., Baleanu, D.: On the generalized fractional derivatives and their Caputo modification. J. Nonlinear Sci. Appl. 10, 2607-2619 (2017)

11. Ramaswamy, M., Shivaji, R.: Multiple positive solutions for classes of $p$-Laplacian equations. Differ. Integral Equ. 17, 1255-1261 (2004)

12. Oruganti, S., Shi, J., Shivaji, R.: Logistic equation with the $p$-Laplacian and constant yield harvesting. Abstr. Appl. Anal. 2004, 723-727 (2004)

13. Ly, I., Seck, D.: Isoperimetric inequality for an interior free boundary problem with $p$-Laplacian operator. Electron. J. Differ. Equ. 287, 12 (2004)

14. Zhi, E., Liu, X., Li, F.: Nonlocal boundary value problem for fractional differential equations with $p$-Laplacian. Math. Methods Appl. Sci. 37, 2651-2662 (2014)

15. Jafari, H., Baleanu, D., Khan, H., Khan, R.A., Khan, A.: Existence criterion for the solutions of fractional order $p$-Laplacian boundary value problems. Bound. Value Probl. 2015(1), 164 (2015)

16. Hu, L., Zhang, S.: On existence results for nonlinear fractional differential equations involving the $p$-Laplacian at resonance. Mediterr. J. Math. 13, 955-966 (2016)

17. Diening, L., Lindqvist, P., Kawohl, B.: Mini-workshop: the $p$-Laplacian operator and applications. Oberwolfach Rep. 10 433-482 (2013)

18. Wang, Y., Hou, C.: Existence of multiple positive solutions for one-dimensional p-Laplacian. J. Math. Anal. Appl. 315 , 144-153 (2006)

19. Al-Mdallal, Q.M., Hajji, M.A.: A convergent algorithm for solving higher-order nonlinear fractional boundary value problems. Fract. Calc. Appl. Anal. 18, 1423-1440 (2015)

20. Abdeljawad, T., Baleanu, D.: On fractional derivatives with exponential kernel and their discrete versions. Rep. Math. Phys. 80, 11-27 (2017)

21. Jankowski, T.: Positive solutions to fractional differential equations involving Stieltjes integral conditions. Appl. Math Comput. 241, 200-213 (2014)

22. Cabada, A., Dimitrijevic, S., Tomovic, T., Aleksic, S.: A Lyapunov approach to the stability of fractional differential equations. Math. Methods Appl. Sci. 40, 1880-1891 (2017)

23. Ntouyas, S.K., Etemad, S.: On the existence of solutions for fractional differential inclusions with sum and integral boundary conditions. Appl. Math. Comput. 266, 235-243 (2015)

24. Günendi, M., Yaslan, I.: Positive solutions of higher-order nonlinear multi-point fractional equations with integra boundary conditions. Fract. Calc. Appl. Anal. 19, 989-1009 (2016)

25. Hu, L., Zhang, S.: Existence results for a coupled system of fractional differential equations with $p$-Laplacian operator and infinite-point boundary conditions. Bound. Value Probl. 2017(1), 88 (2017) 
26. Ali, A., Samet, B., Shah, K., Khan, R.A.: Existence and stability of solution to a toppled systems of differential equations of non-integer order. Bound. Value Probl. 2017(1), 16 (2017)

27. Agarwal, R., O'Regan, D., Hristova, S.: Stability of Caputo fractional differential equations by Lyapunov functions. Appl. Math. 60, 653-676 (2015)

28. Wang, J.: Stability of noninstantaneous impulsive evolution equations. Appl. Math. Lett. 73, 153-162 (2011)

29. Ulam, S.: Problems in Modern Mathematics. Courier Corporation (2004)

30. Khan, H., Tunc, C., Chen, W., Khan, A.: Existence theorem and Hyers-Ulam stability for a class of hybrid fractional differential equation with p-Laplacian operator. J. Appl. Anal. Comput. 8, 1211-1226 (2018)

31. Jung, S.: Hyers-Ulam stability of a system of first order linear differential equations with constant coefficients. J. Math. Anal. Appl. 320, 549-561 (2006)

32. Jung, S.M., Rassias, T.M.: Generalized Hyers-Ulam stability of Riccati differential equation. J. Math. Anal. Appl. 11, 777-782 (2008)

33. Trigeassou, J., Maamri, N., Sabatier, J., Oustaloup, A.: A Lyapunov approach to the stability of fractional differential equations. Signal Process. 91, 437-445 (2011)

34. Isaia, F.: On a nonlinear integral equation without compactness. Acta Math. Univ. Comen. 75(2), 233-240 (2006)

35. Deimling, K.: Nonlinear Functional Analysis. Courier Corporation (2010)

36. Podlubny, l.: Fractional Differential Equations. Academic Press, New York (1999)

37. Shen, T., Liu, W., Shen, X.: Existence and uniqueness of solutions for several BVPs of fractional differential equations with $p$-Laplacian operator. Mediterr. J. Math. 13, 4623-4637 (2016)

\section{Submit your manuscript to a SpringerOpen ${ }^{\circ}$ journal and benefit from:}

- Convenient online submission

- Rigorous peer review

Open access: articles freely available online

High visibility within the field

Retaining the copyright to your article

Submit your next manuscript at $\mathbf{s p r i n g e r o p e n . c o m ~}$ 